\title{
Optimization Based Partitioning Selection for Improved Contaminant Detection Performance
}

\author{
Alexis Kyriacou ${ }^{1}$, Stelios Timotheou ${ }^{1}$, Vasso Reppa ${ }^{1}$, Francesca Boem ${ }^{2}$, Christos Panayiotou ${ }^{1}$ \\ Marios Polycarpou ${ }^{1}$ and Thomas Parisini ${ }^{3}$
}

\begin{abstract}
Indoor Air Quality monitoring is an essential ingredient of intelligent buildings. The release of various airborne contaminants into the buildings, compromises the health and safety of occupants. Therefore, early contaminant detection is of paramount importance for the timely activation of proper contingency plans in order to minimize the impact of contaminants on occupants health. The objective of this work is to enhance the performance of a distributed contaminant detection methodology, in terms of the minimum detectable contaminant release rates, by considering the joint problem of partitioning selection and observer gain design. Towards this direction, a detectability analysis is performed to derive appropriate conditions for the minimum guaranteed detectable contaminant release rate for specific partitioning configuration and observer gains. The derived detectability conditions are then exploited to formulate and solve an optimization problem for jointly selecting the partitioning configuration and observer gains that yield the best contaminant detection performance.
\end{abstract}

\section{INTRODUCTION}

Intelligent buildings improve the comfort and productivity of occupants and ensure their health and safety by monitoring and controlling the building environment [1]. Indoor Air Quality (IAQ) has been identified as one of the three most important factors (apart from visual and thermal comfort) that influence the quality of occupants' life [2]. However, IAQ is often at risk by various airborne contaminants [3]. Given these safety-compromising conditions, the prompt detection of contaminant events and the isolation of the contaminant source location are of paramount importance to ensure people's safety.

A building can be considered as a system of interconnected elements (zones) interacting through airflows, which can potentially transport air contaminants between them.

This work was partially supported by the European Research Council (ERC) through the FAULT-ADAPTIVE Project under the ERC Advanced Grant and by the European Unions Horizon 2020 research and innovation program under grant agreement No 739551 (KIOS CoE).

${ }^{1}$ A. Kyriacou, S. Timotheou, V. Reppa, C. Panayiotou and M. Polycarpou are with the KIOS Research and Innovation Center of Excellence and the Department of Electrical and Computer Engineering, University of Cyprus, Nicosia, Cyprus ( $\{$ akyria09, timotheou.stelios\}eucy.ac.cy;

reppavassolgmail.com; \{christosp,mpolycar\}

(ucy.ac.cy).

${ }^{2} \mathrm{~F}$. Boem is with the Dept. of Electronic and Electrical Engineering at University College London, UK. (francesca.l.boemegmail.com)

${ }^{3}$ T. Parisini is with the Dept. of Electrical and Electronic Engineering at the Imperial College London, UK, with the KIOS Research and Innovation Center of Excellence, University of Cyprus, and also with the Dept. of Engineering and Architecture at University of Trieste, Italy. (t.parisiniegmail.com)
The contaminant existence in the system can be modeled as a fault, enabling the use of fault diagnosis tools for detecting and isolating a contaminant source. In our previous works [4], [5], we have developed both a centralized and a distributed state-space model for describing the contaminant dispersion in the building interior along with the development of Contaminant Detection and Isolation (CDI) algorithms. The building partitioning problem has also been considered in [5] for defining the individual subsystems for the distributed CDI algorithms. The partitioning approach in [5] develops a mathematical programming approach that groups the building zones into individual subsystems while minimizes the interconnection uncertainty (in terms of airflow influence) between the contaminant detection agents and creates partitions of similar size. In the areas of distributed system monitoring and control approaches, considering system partitioning from the system design phase has been shown to decrease the complexity and improve the performance of the approach [6]-[8].

In this work, we aim at improving the contaminant detection performance through the selection of the best partitioning solution that enables the detection of the smallest contaminant release rates. The influence of the subsystem configuration to the possible detectable contaminant release rate is defined based on the detectability analysis of the CDI approach. Specifically, the analysis shows the effects of a contaminant source on the residuals and adaptive thresholds and expresses the contaminant detection capabilities, in terms of minimum detectable release rate and observer gains [9]. In order to enable the comparison of all possible partitioning solutions, an optimization algorithm is developed for extracting the observer gains that give the minimum detectable contaminant release rate in every zone, similar to [10], where an optimization methodology was developed for the design of a centralized observer-based, sensor fault detection scheme for a class of nonlinear systems. The optimization algorithm proposed in this work considers two different objectives: (a) minimize the sum of the contaminant release rates of the zones with the worst detection performance in each subsystem (among the zones of each subsystem), and (b) minimize the sum of the detectable contaminant release rates in all building zones.

In summary, the main contributions of this work are:

1) The modeling of the impact of partitioning on the ability of a CDI scheme to detect contaminants.

2) The formulation of a Semi-Definite Programming (SDP) optimization problem for the selection of ob- 
server gains for a specific system partitioning.

3) The selection of the partitioning solution that has the best contaminant detection performance.

The paper is organized as follows. Section II introduces the system model and the problem of interest. Section III describes a distributed contaminant detection scheme, while Section IV performs the detectability analysis which quantifies the lowest detectable contaminant release rate for a given partitioning solution. Section V develops an optimization approach for extracting the best observer gains and partitioning solution. Section VI examines the effectiveness of the proposed approach for a real building model, while Section VII provides concluding remarks and future directions.

Notation: The symbol $|\cdot|$ denotes the absolute value for scalars, the Euclidean norm $\left(\ell_{2}\right.$-norm) for matrices and vectors, and the cardinality for sets, if not explicitly stated otherwise. Sets are represented with calligraphic letters and the identity matrix with the symbol $\mathbb{I}$.

\section{System MODEL}

Given a $n$-zone building, a matrix $A \in \mathbb{R}^{n \times n}$ with elements $a^{(i, j)}$ can be used to describe the dependencies between the zones, mainly as a result of the airflows between zones. An element $a^{(i, j)}$ is non zero only if there is a leakage path (door, window, HVAC ducts, etc.) between zone $i$ and zone $j$. The distributed model describing the contaminant dispersion in each of $\mathrm{K}$ interconnected subsystems $\Sigma_{I}, I \in \mathscr{K}=[1, \ldots, K]$, with $n_{I}$ zones resulting by a system partitioning is:

$$
\begin{aligned}
\Sigma_{I}: \quad \dot{x}_{I}(t)= & \left(A_{I}+\Delta A_{I}\right) x_{I}(t)+Q_{I}^{-1} B_{I} u_{I}(t)+Q_{I}^{-1} G_{I} g_{I}(t) \\
& +\left(H_{I}+\Delta H_{I}\right) z_{I}(t), \\
y_{I}(t)= & C_{I} x_{I}(t)+w_{I}(t),
\end{aligned}
$$

where $x_{I} \in \mathbb{R}^{n_{I}}$ represents the contaminant concentration of all building zones in $\Sigma_{I}\left(x_{I}^{(j)} \in \mathbb{R}\right.$, denotes the concentration in the $j$-th zone of $\Sigma_{I}$ ), variables $u_{I} \in \mathbb{R}^{p_{I}}$, represent the controllable inputs in the form of doors, windows, fans and air handling units, while $B_{I} \in \mathbb{B}^{n_{I} \times p_{I}}$ is a zone index matrix concerning their locations in $\Sigma_{I}$, with $\mathbb{B}=\{0,1\}$. Interconnection variables $z_{I} \in \mathbb{R}^{l_{I}}$ refer to the state of the neighboring subsystems having a direct connection with $\Sigma_{I}$ i.e. $z_{I}$ is made up of states of $x_{I}$ that belongs to subsystems different from $\Sigma_{I}$. The local contaminant source and its location are represented by terms $g_{I} \in \mathbb{R}^{n_{I}}$ and $G_{I} \in \mathbb{B}^{n_{I} \times n_{I}}$ respectively. Note that $Q_{I} \in \mathbb{R}^{n_{I} \times n_{I}}$ is a diagonal matrix, i.e. $Q_{I}=\operatorname{diag}\left(q_{I}^{(1)}, q_{I}^{(2)}, \ldots, q_{I}^{\left(n_{I}\right)}\right)$ where $q_{I}^{(j)}$ is the volume of the $j$-th zone in $\Sigma_{I}$. It is also worth noting that the existence of a maximum of one contaminant source per subsystem at a time is considered while the term $g_{I}(t)$, is equivalent to process faults that impact the normal system operation. Vector $y_{I} \in \mathbb{R}^{m_{I}}$ in (2), represents the contaminant concentration as measured by the sensors monitoring $\Sigma_{I}, C_{I} \in \mathbb{B}^{m_{I} \times n_{I}}$ is a zone index matrix specifying the sensors' locations in $\Sigma_{I}$ and $w_{I} \in \mathbb{R}^{m_{I}}$ represents an additive measurement noise. Finally matrices $A_{I}, H_{I}$ are sub-matrices of matrix $A$ while matrices $\Delta A_{I}$ and $\Delta H_{I}$ collectively account for the presence of modeling and interconnection uncertainty of $\Sigma_{I}$ as a result of disturbances due to variable wind speed, wind direction and leakage openings.

\section{Distributed Contaminant Detection}

In this section, a methodology for detecting multiple contaminant sources is presented. For each subsystem $\Sigma_{I}$, a contaminant detector agent denoted by $\mathscr{D}_{I}$ is responsible for detecting a possible contaminant source. The agents are allowed to exchange measurement information with their neighboring agents, defined as the agents that share physical interconnections represented by the terms $H_{I}, z_{I}$. The design of contaminant detectors $\left(\mathscr{D}_{I}\right)$ relies on comparing residual signals to corresponding adaptive thresholds.

\section{A. Contaminant Event Detection}

1) Residual Generation: The estimation model of $\mathscr{D}_{I}$ is computed by selecting the following observer:

$\dot{\hat{x}}_{I}(t)=A_{I} \hat{x}_{I}(t)+Q_{I}^{-1} B_{I} u_{I}(t)+H_{I} y_{z_{I}}(t)+L_{I}\left(y_{I}(t)-C_{I} \hat{x}_{I}(t)\right)$,

where $\hat{x}_{I} \in \mathbb{R}^{n_{I}}$ is the estimation of $x_{I}$ with $\hat{x}_{I}(0)=0$, $L_{I} \in \mathbb{R}^{n_{I} \times m_{I}}$ is the observer gain matrix (the design of $L_{I}$ will be discussed in Section V) and $y_{z_{I}}(t)=z_{I}(t)+w_{z_{I}}(t)$ is the transmitted sensor information, where $w_{z_{I}} \in \mathbb{R}^{\ell_{I}}$ is the corresponding measurement noise vector.

The $k$-th residual of the detector $\mathscr{D}_{I}$, is denoted by $\varepsilon_{y_{I}}^{(k)} \in \mathbb{R}$ and is defined as:

$$
\varepsilon_{y_{I}}^{(k)}(t)=C_{I}^{(k)} \varepsilon_{x_{I}}(t)+w_{I}^{(k)}(t)
$$

where $C_{I}^{(k)}$ is the $k$-th row of $C_{I}, k \in\left\{1, \ldots, m_{I}\right\}$ and vector $\varepsilon_{x_{I}}(t) \triangleq x_{I}(t)-\hat{x}_{I}(t)$ represent the state estimation error.

2) Computation of Adaptive Thresholds: The $k$-th adaptive threshold of $\mathscr{D}_{I}$, denoted by $\bar{\varepsilon}_{y_{I}}^{(k)}(t)$, is designed to consider modeling uncertainties $\Delta A_{I}, \Delta H_{I}$ and sensor noise $w_{I}, w_{z_{I}}$ to ensure robustness of the detector $\mathscr{D}_{I}$. The modeling uncertainty and the noise corrupting the measurements of each sensor, are assumed unknown but uniformly bounded for all $I \in\{1, \ldots, K\}, j \in\left\{1, \ldots, \ell_{I}\right\}$ and $k \in\left\{1, \ldots, m_{I}\right\}$ :

$$
\begin{array}{rlrl}
\left|\Delta A_{I}\right| & \leq \overline{\Delta A}_{I}, & \left|\Delta H_{I}\right| & \leq \overline{\Delta H}_{I}, \\
\left|w_{I}^{(k)}(t)\right| & \leq \bar{w}_{I}^{(k)} & \left|w_{z I}^{(j)}(t)\right| \leq \bar{w}_{z I}^{(j)} .
\end{array}
$$

Under healthy conditions (i.e., $g_{I}(t)=0$ ), the state estimation error is denoted by:

$$
\dot{\varepsilon}_{x_{I}}(t)=A_{L_{I}} \varepsilon_{x_{I}}(t)+\Delta H_{I} z_{I}(t)+\Delta A_{I} x_{I}(t)-H_{I} w_{z_{I}}(t)-L_{I} w_{I},
$$

where, $A_{L_{I}}=A_{I}-L_{I} C_{I}$ and the bound of its solution can be calculated as:

$$
\begin{gathered}
\bar{\varepsilon}_{x_{I}}(t) \triangleq E_{1}(t)+\int_{0}^{t} \rho_{I} e^{-\left(\xi_{I}-\rho_{I} \overline{\Delta A}_{I}\right)(t-\tau)} E_{1}(\tau) d \tau \\
E_{1}(t)=\rho_{I} e^{-\xi_{I} t} \bar{x}_{I}+\frac{\rho_{I}}{\xi_{I}}\left(\left(\left|H_{I}\right|+\overline{\Delta H}_{I}\right) \bar{w}_{z_{I}}+\left|L_{I}\right| \bar{w}_{I}\right)\left(1-e^{-\xi_{I} t}\right) \\
+\int_{0}^{t} \rho_{I} e^{-\xi_{I}(t-\tau)}\left(\overline{\Delta H}_{I}\left|y_{z_{I}}(\tau)\right|+\overline{\Delta A}_{I}\left|\hat{x}_{I}(\tau)\right|\right) d \tau
\end{gathered}
$$


where $\bar{x}_{I} \in \mathbb{R}$ is a known bound satisfying $\left|\varepsilon_{x_{I}}(0)\right| \leq \bar{x}_{I}$ and $\rho_{I}, \xi_{I}$ are positive constants selected such that $\left|e^{A_{L_{I}} t}\right| \leq$ $\rho_{I} e^{-\xi_{I} t}$, for all $t$ and $\xi_{I}>\rho_{I} \overline{\Delta A}_{I}$.

The $k$-th adaptive threshold of the detector $\mathscr{D}_{I}$ bounding the residual under healthy conditions is described by:

$$
\begin{aligned}
\bar{\varepsilon}_{y_{I}}^{(k)}(t)= & \frac{\alpha_{I}^{(k)}}{\zeta_{I}^{(k)}}\left(\left(\left|H_{I}\right|+\overline{\Delta H}_{I}\right) \bar{w}_{z_{I}}+\left|L_{I}\right| \bar{w}_{I}\right)\left(1-e^{-\zeta_{I}^{(k)} t}\right) \\
& +\alpha_{I}^{(k)} e^{-\zeta_{I}^{(k)} t} \bar{x}_{I}+\int_{0}^{t} \alpha_{I}^{(k)} e^{-\zeta_{I}^{(k)}(t-\tau)}\left(\overline{\Delta H}_{I}\left|y_{z_{I}}(\tau)\right|\right. \\
& \left.+\overline{\Delta A}_{I}\left|\hat{x}_{I}(\tau)\right|+\overline{\Delta A}_{I} \bar{\varepsilon}_{x_{I}}(\tau)\right) d \tau+\bar{w}_{I}^{(k)}
\end{aligned}
$$

where $\alpha_{I}^{(k)}, \zeta_{I}^{(k)}$ are positive constants chosen such that $\left|C_{I}^{(k)} e^{A_{L_{I}} t}\right| \leq \alpha_{I}^{(k)} e^{-\zeta_{I}^{(k)} t}$, for all $t$. A detailed description of the bound derivation process can be found in [5]. The $k$-th adaptive threshold in (10) of the detector $\mathscr{D}_{I}$ is designed to bound the residual under healthy conditions such that:

$$
\left|\varepsilon_{y_{I}}^{(k)}(t)\right| \leq \bar{\varepsilon}_{y_{I}}^{(k)}(t) .
$$

3) Detection Decision Logic: If there is a time instant $t$ at which for at least one $k \in\left\{1, \ldots, m_{I}\right\}$

$$
\left|\varepsilon_{y_{I}}^{(k)}(t)\right|>\bar{\varepsilon}_{y_{I}}^{(k)}(t),
$$

then the detector $\mathscr{D}_{I}$ infers the presence of a contaminant source in subsystem $\Sigma_{I}, I \in\{1, \ldots, K\}$.

\section{DETECTABILITY ANALYSIS}

In the previous section, it was shown that if the residual error exceeds the threshold then a contaminant source is present. In this section, we derive the conditions which must be satisfied in order for a contaminant to be detected in a zone. The detectability analysis aims to isolate the variables that affect the contaminant detection performance [9]. Specifically, the goal is to assess and quantify the impact of partitioning solutions through the detectability conditions. This information will later be used in order to formulate an optimization algorithm to define the best possible partitioning solution and the observer gains in order to ensure high contaminant detection performance.

First, let us define the time instant $T_{R}$ as the time that a contaminant is released anywhere in the system:

$$
T_{R}=\min _{t} \bigcup_{I \in\{1, \ldots, K\}}\left\{\min _{t}\left\{t:\left|g_{I}(t)\right|>0\right\}\right\} .
$$

For $t \geq T_{R}$, the state vector $x_{I}$ and the state estimation vector provided by the observer described in (3), can be separated into healthy and faulty dynamics as $x_{I}(t)=x_{I H}(t)+$ $x_{I F}(t)$ and $\hat{x}_{I}(t)=\hat{x}_{I H}(t)+\hat{x}_{I F}(t)$ respectively, where $x_{I H}$ and $\hat{x}_{I H}$ correspond to the state and state estimation vectors assuming $g_{I}(t)=0$. In turn, the estimation error for $t \geq T_{R}$ can be written as $\varepsilon_{x_{I}}(t)=\varepsilon_{x_{I H}}(t)+\varepsilon_{x_{I F}}(t)$ where $\varepsilon_{x_{I H}}(t)$ is the solution of (7), where $x_{I}(t)=x_{I H}(t)$. Using (4), the $k$-th residual of the detector $\mathscr{D}_{I}$ for $t \geq T_{R}$ can be expressed as:

$$
\varepsilon_{y_{I}}^{(k)}(t)=C_{I}^{(k)}\left(\varepsilon_{x_{I H}}(t)+\varepsilon_{x_{I F}}(t)\right)+w_{I}^{(k)}=\varepsilon_{y_{I H}}^{(k)}(t)+\varepsilon_{y_{I F}}^{(k)}(t)
$$

where the term $\varepsilon_{y_{I F}}^{(k)}(t)$ represents the effects of a contaminant release on the residual and is given by:

$$
\varepsilon_{y_{I F}}^{(k)}(t)=\int_{T_{R}}^{t} C_{I}^{(k)} e^{A_{L_{I}}(t-\tau)}\left(\Delta A_{I} x_{I F}(\tau)+Q_{I}^{-1} G_{I} g_{I}(\tau)\right) d \tau
$$

The $k$-th adaptive threshold in (10) is affected by the contaminant release in the system and for $t \geq T_{R}$ can be expressed as $\bar{\varepsilon}_{y_{I}}^{(k)}(t)=\bar{\varepsilon}_{y_{I H}}^{(k)}(t)+\bar{\varepsilon}_{y_{I F}}^{(k)}(t)$ where $\bar{\varepsilon}_{y_{I H}}^{(k)}$ is given using $\bar{\varepsilon}_{x_{I}}=\bar{\varepsilon}_{x_{I H}}$ and $\hat{x}_{I}=\hat{x}_{I H}$ in (10). It is important to mention that $\bar{\varepsilon}_{y_{I H}}^{(k)}$ is a time-varying threshold bounding the effects of modeling uncertainties and noise, $\left|\varepsilon_{y_{I H}}^{(k)}(t)\right| \leq \bar{\varepsilon}_{y_{I H}}^{(k)}(t)$ for all t. Finally, the effect of the contaminant release on the $k$-th threshold of detector $\mathscr{D}_{I}$ is derived as:

$$
\bar{\varepsilon}_{y_{I F}}^{(k)}(t)=\int_{T_{R}}^{t} a_{I}^{(k)} e^{-\zeta_{I}^{(k)}(t-\tau)} \overline{\Delta A}_{I}\left(\bar{\varepsilon}_{x_{I F}}(\tau)+\left|\hat{x}_{I F}(\tau)\right|\right) d \tau
$$

Remark 4.1: If there is zero uncertainty (i.e., $\Delta A_{I}=$ $\left.\Delta H_{I}=0\right)$ then the threshold in (10) is not affected by the contaminant release (i.e., $\bar{\varepsilon}_{y_{I F}}^{(k)}(t)=0$ ).

Theorem 4.2: If there exist a time instant $t^{*}>T_{R}$ such that the contaminant source $g_{I}$ satisfies the condition

$$
\left|\varepsilon_{y_{I F}}^{(k)}\left(t^{*}\right)\right|-\bar{\varepsilon}_{y_{I F}}^{(k)}\left(t^{*}\right)>2 \bar{\varepsilon}_{y_{I H}}^{(k)}\left(t^{*}\right),
$$

for at least one $k \in\left[1, \ldots, m_{I}\right]$, the presence of contaminant in subsystem $\Sigma_{I}$ is guaranteed to be detected.

Proof: Using (14), the following bound can be derived as:

$$
\left|\varepsilon_{y_{I}}^{(k)}\left(t^{*}\right)\right| \geq\left|\varepsilon_{y_{I F}}^{(k)}\left(t^{*}\right)\right|-\left|\varepsilon_{y_{I H}}^{(k)}\left(t^{*}\right)\right| .
$$

Given that $\left|\varepsilon_{y_{I H}}^{(k)}\right| \leq \bar{\varepsilon}_{y_{I H}}^{(k)}$, we obtain

$$
\left|\varepsilon_{y_{I}}^{(k)}\left(t^{*}\right)\right| \geq\left|\varepsilon_{y_{I F}}^{(k)}\left(t^{*}\right)\right|-\bar{\varepsilon}_{y_{I H}}^{(k)}\left(t^{*}\right) .
$$

Combining (17) and (19) yields

$$
\left|\varepsilon_{y_{I}}^{(k)}\left(t^{*}\right)\right|>2 \bar{\varepsilon}_{y_{I H}}^{(k)}\left(t^{*}\right)+\bar{\varepsilon}_{y_{I F}}^{(k)}\left(t^{*}\right)-\bar{\varepsilon}_{y_{I H}}^{(k)}\left(t^{*}\right)=\bar{\varepsilon}_{y_{I}}^{(k)}\left(t^{*}\right),
$$

which based on (12) ensures that the presence of contaminant in $\Sigma_{I}$ is detected at time instant $t^{*}$.

\section{A. Partitioning Effect on Detection Performance}

The detectability bound presented in (17), characterizes the ability of a detector $\mathscr{D}_{I}$ to detect the presence of a contaminant in each zone of $\Sigma_{I}$. This ability is expressed by the relationship of the contaminant $g_{I}(t)$ with the computed adaptive thresholds. It is important to note that the detectable contaminants are expressed considering worstcase detectability conditions. However, it does not provide a clear indication on how the bound is affected by the system partitioning, since for each selected partitioning solution, variable selections (i.e., observer gains and positive constants $\zeta_{I}, \alpha_{I}$ used in the threshold calculation) change, to follow stability and convergence constraint satisfiability.

Towards simplification of the detectability bound the following assumptions have been considered: 
1) There is a maximum of one contaminant source (e.g., in $\Sigma_{I}$ ) with constant release rate indicated by $\bar{g}_{I}^{(k)}$,

2) All states are measurable $C_{I}=\mathbb{I}$,

3) There is zero modeling uncertainty $\Delta A_{I}=0, \Delta H_{I}=0$ and zero initial concentration $x_{I}(0)=0$.

Constant contaminant source and zero modeling uncertainty assumptions allow to extract the solution of the left part of inequality (17) and the detectability bound for the $k$-th zone of subsystem $\Sigma_{I}$ is simplified to:

$$
\bar{g}_{I}^{(k)}>\frac{2 \bar{\varepsilon}_{y_{I H}}^{(k)}(t)}{\left|C_{I}^{(k)} e^{A_{L_{I}} t} A_{L_{I}}^{-1}\left(e^{-A_{L_{I}} T_{R}}-e^{-A_{L_{I}} t}\right) Q_{I}^{-1} G_{I(k)}\right|},
$$

where $G_{I(k)}$ is the $k$-th column of matrix $G_{I}$.

The measurable states allow the observer gain matrix $L_{I}$ to be chosen such that matrix $A_{L_{I}}=\left(A_{I}-L_{I} C_{I}\right)$ is diagonal and the detectability bound (20) can be further simplified into:

$$
\bar{g}_{I}^{(k)}>\frac{2 \bar{\varepsilon}_{y_{I H}}^{(k)}(t)\left|A_{L_{I}}^{(k, k)} q_{I}^{(k, k)}\right|}{\left|e^{A_{L_{I}}^{(k, k)}\left(t-T_{R}\right)}-1\right|},
$$

where $A_{L_{I}}^{(k, k)}$ and $q_{I}^{(k, k)}$ indicate the $k$-th diagonal elements of matrices $A_{L_{I}}$ and $Q_{I}$ respectively. Note that by considering full sensor measurements, the positive constants defined for (10) can be selected as $\zeta_{I}^{(k)}=\left|A_{L_{I}}{ }^{(k, k)}\right|$ and $\alpha_{I}^{(k)}=1$ for all $I \in \mathscr{K}, k \in\left[1, \ldots, n_{I}\right]$.

Using the assumptions of zero model uncertainty and zero contaminant concentration under healthy conditions, $\bar{\varepsilon}_{y_{I H}}^{(k)}$ becomes:

$$
\bar{\varepsilon}_{y_{I H}}^{(k)}(t)=\left(\bar{w}_{z_{I}}\left|H_{I}\right|+\left|L_{I}\right| \bar{w}_{I}\right)\left(\frac{1-e^{-\left|A_{L_{I}}^{(k, k)}\right| t}}{\left|A_{L_{I}}^{(k, k)}\right|}\right)+\bar{w}_{I}^{(k)}
$$

In steady-state conditions, terms $e^{A_{L_{I}}^{(k, k)\left(t-T_{R}\right)}}$ and $e^{-\left|A_{L_{I}}^{(k, k)}\right| t}$ of (21) and (22) tend to zero, so that (21) becomes:

$$
\bar{g}_{I}^{(k)}>2\left|q_{I}^{(k, k)}\right|\left(\bar{w}_{z_{I}}\left|H_{I}\right|+\left|L_{I}\right| \bar{w}_{I}+\bar{w}_{I}^{(k)}\left|A_{L_{I}}^{(k, k)}\right|\right) .
$$

Condition (23) indicates that the detectability performance of different partitioning solutions depends on the observer gains. Therefore, in the next section we develop an optimization algorithm for designing the observer gains and selecting the partitioning solution that results in the best detectability performance.

\section{Detectability Bound Optimization}

To solve the joint partitioning and observer gain selection problem the approach taken is to consider all possible partitioning configurations that satisfy connectivity (i.e., every pair of zones in the same subsystems is connected through at least one airflow path) and size constraints (minimum number of 2 zones per subsystem), and optimally solve the observer gain selection problem.

Given a partitioning solution as a set $\mathscr{V}=\cup_{h \in \mathscr{K}} \mathscr{V}_{h}$, that hold the global indices of zones included in each $\Sigma_{h}, h \in \mathscr{K}$ and matrix $A$ that describes the dependencies between the zones, one can define the following constant quantities:

$$
\begin{aligned}
& A_{a d j}^{(i, j)}=\left\{\begin{array}{l}
1, \text { if } a^{(i, j)} \neq 0 \\
0, \text { if } a^{(i, j)}=0
\end{array} \quad i, j \in\{1, \ldots, n\},\right. \\
& l_{h}=\sum_{\substack{i \in \mathscr{V}_{h} \\
j \notin \mathscr{V}_{h}}} \frac{A_{a d j}^{(i, j)}}{\sum_{i \in \mathscr{V}_{h}} A_{a d j}^{(i, j)}}, \quad h \in \mathscr{K} \\
& \bar{w}_{h}=\bar{w} \sqrt{\left|\mathscr{V}_{h}\right|} \quad h \in \mathscr{K} \\
& \bar{w}_{z_{h}}=\bar{w} \sqrt{l_{h}} \quad h \in \mathscr{K} \\
& H_{h}^{(i, j)}=\left\{\begin{array}{l}
a_{h}^{(i, j)} \\
0
\end{array}\right. \\
& j \in \mathscr{V}_{h}, i \notin \mathscr{V}_{h}, h \in \mathscr{K} \\
& \text { otherwise } \\
& \overline{H_{h}}=\left|H_{h}\right| \\
& h \in \mathscr{K}
\end{aligned}
$$

Note that we have assumed without loss of generality the same sensor noise bounds, $\bar{w}$, for all sensors.

\section{A. Optimization Formulation}

The formulation optimizes the observer gains for a given partitioning solution in order to minimize the detectable contaminant release rate, i.e. to satisfy condition (23) for the smallest possible contaminant release rates. The observer gains are also constrained to guarantee convergence for the designed observer-based estimators (i.e., matrix $A_{L_{I}}$ is stable). The problem can be formulated using different objective functions depending on the specific application needs. In this paper we utilize the objectives

$$
\begin{array}{ll}
\text { Obj. } 1: & \min _{\beta, \lambda} \sum_{i \in \mathscr{V}} g_{i}^{z}, \\
\text { Obj. } 2: & \min _{\beta, \lambda} \sum_{h \in \mathscr{K}} g_{h}^{p},
\end{array}
$$

where variable $g_{i}^{z}$ shows the resulting minimum detectable contaminant release rate in each zone $i=[1, \ldots, n]$, while variable $g_{h}^{p}=\max _{i \in \mathscr{V}_{h}}\left\{g_{i}^{z}\right\}, h \in \mathscr{K}$ indicates the contaminant release rate of the zone with the worst detection performance in subsystem $h \in \mathscr{K}$. Obj. 1 minimizes the sum of detectable contaminant release rates in all zones of the building, while Obj. 2 only minimizes the sum of the contaminant release rates of the zones with the worst detection performance in each subsystem.

Given subsystem configuration $\mathscr{V}_{h}, h \in \mathscr{K}$ and its corresponding subsystem noise bound $\bar{w}_{h}$, interconnection noise bound $\bar{w}_{z_{h}}$ and interconnection bound $\bar{H}_{h}$ calculated through equations (26), (27) and (29), constraints (32) ensure satisfaction of the detectability condition (23). In particular, constraints $(32 \mathrm{a})-(32 \mathrm{c})$ are used to define gain matrix $L_{h}$ of each subsystem. Inequality (32b) ensures observer stability since $a^{(i, i)}-\lambda^{(i)} \leq-\bar{\omega}<0$ where $\bar{\omega}>0$ a small positive value. The $\ell_{2}$-norm of matrix $L_{h}$ is nonlinear (but convex) and equates to finding the maximum singular value of the matrix. In fact, the Linear Matrix Inequality (LMI) (32c) ensures that $\beta_{h}=\left|L_{h}\right|$ [11]. The reason is that condition (23) requires $\beta_{h}$ to take the minimum possible value which is achieved when the LMI is satisfied with equality. Combining (6), (26), (27), (29) and constraints (32a) - (32c), the 


$$
\begin{aligned}
& \text { Constraints defining detectability condition (23) }
\end{aligned}
$$

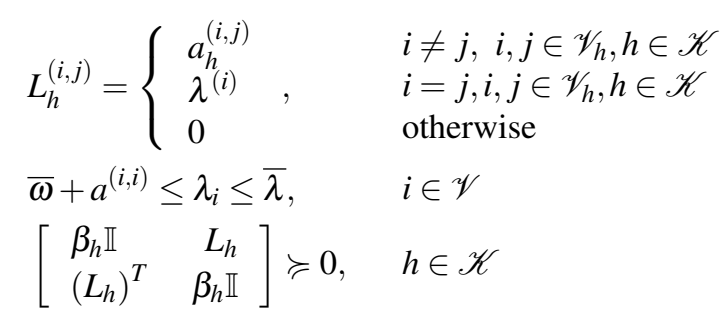

$$
\begin{aligned}
& g_{i}^{z} \geq 2 q^{(i)}\left(\overline{w_{z_{h}}} \overline{H_{h}}+\overline{w_{h}} \beta_{h}+\bar{w}\left(a^{(i, i)}-\lambda^{(i)}\right)\right), \quad i \in \mathscr{V}_{h}, h \in \mathscr{K} \\
& g_{i}^{z} \geq 2 q^{(i)}\left(\overline{w_{z_{h}}} \overline{H_{h}}+\overline{w_{h}} \beta_{h}-\bar{w}\left(a^{(i, i)}-\lambda^{(i)}\right)\right), \quad i \in \mathscr{V}_{h}, h \in \mathscr{K}
\end{aligned}
$$

detectability constraint is defined in (32d) and (32e). The maximization term in $g_{h}^{p}, h \in \mathscr{K}$, in relation to Obj. 2 is eliminated through the introduction of the constraint

$$
g_{h}^{p} \geq g_{i}^{z}, i \in \mathscr{V}_{h}, h \in \mathscr{K} .
$$

In summary, the resulting optimization problems from Obj. 1 and Obj. 2 are the following:

$$
\begin{array}{cl}
\text { OP1: } & \min _{\beta, \lambda, g^{z}} \sum_{i \in \mathscr{V}} g_{i}^{z} \\
\text { s.t. } & \text { Constraints }(32) \\
\text { OP2: } & \min _{\beta, \lambda, g^{z}, g^{p}} \sum_{h \in \mathscr{K}} g_{h}^{p}
\end{array}
$$$$
\text { s.t. Constraints (32) and (33). }
$$

Optimization problem OP1 finds the best observer gains $L_{h}^{*}$ and the minimum detectable contaminant release rate in each zone $i$ is indicated by $g_{i}^{z *}$. Similarly, OP2 results in observer gains $L_{h}^{*}$ and the minimized maximum detectable contaminant release rate $g_{h}^{p *}$ in each subsystem $\Sigma_{h}$. OP1 and OP2 are both linear Semi-definite Programming (SDP) formulations which are convex, and hence they can be optimally solved with standard SDP solvers.

Optimization of observer gains enables the comparison between all possible partitioning configurations in terms of the solutions from OP1 and OP2, for jointly selecting the partitioning configuration and observer gains that result in the best detection performance.

\section{Vi. Performance Evaluation}

In this section, we evaluate the effectiveness of the developed optimization approach by conducting simulations on a real building model of Holmes house ${ }^{1}$. The building is comprised of 14 zones (i.e., Z1, ..,Z14) and 30 leakage path openings. For the examined scenario, it is assumed that an ambient wind of speed $10 \mathrm{~m} / \mathrm{s}$ blowing from the east is the cause of air movement in the building interior. The resulting airflows are calculated using the CONTAM [13] software and the corresponding matrix A appears in Fig. 1.

\footnotetext{
${ }^{1}$ Holmes house refers to a low-rise residential house model scaled up for Simulating Airflow and Contaminant Transport in buildings [12].
}

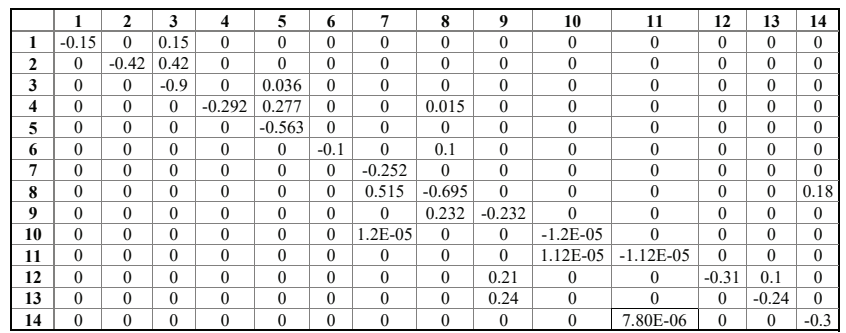

Fig. 1. Matrix A for the Holmes building.

TABLE I

POSSIBLE PARTITIONING OF HOLMES HOUSE FOR $K=2$ SUBSYSTEMS

\begin{tabular}{|c|c|c|}
\hline$P_{n_{K}}$ & Subsystem 1 $\left(\mathscr{V}_{1}\right)$ & Subsystem 2 $\left(\mathscr{V}_{2}\right)$ \\
\hline$P_{1}$ & {$[1,2,3,4,5,6,7,8,9,10,11,14]$} & {$[12,13]$} \\
\hline$P_{2}$ & {$[1,2,3,4,5,6,7,8,9,10,12,13]$} & {$[11,14]$} \\
\hline$P_{3}$ & {$[1,2,3,4,5,6,7,8,9,12,13,14]$} & {$[10,11]$} \\
\hline$P_{4}$ & {$[1,2,3,4,5,6,7,8,9,12,13]$} & {$[10,11,14]$} \\
\hline$P_{5}$ & {$[1,2,3,4,5,6,7,8,10,11,14]$} & {$[9,12,13]$} \\
\hline$P_{6}$ & {$[1,2,3,4,5,6,8,9,11,12,13,14]$} & {$[7,10]$} \\
\hline$P_{7}$ & {$[1,2,3,4,5,6,8,9,12,13,14]$} & {$[7,10,11]$} \\
\hline$P_{8}$ & {$[1,2,3,4,5,6,8,9,12,13]$} & {$[7,10,11,14]$} \\
\hline$P_{9}$ & {$[1,2,3,4,5]$} & {$[6,7,8,9,10,11,12,13,14]$} \\
\hline$P_{10}$ & {$[1,2,3,5]$} & {$[4,6,7,8,9,10,11,12,13,14]$} \\
\hline$P_{11}$ & {$[1,2,3]$} & {$[4,5,6,7,8,9,10,11,12,13,14]$} \\
\hline
\end{tabular}

All feasible partitioning configuration for $K=2$ subsystems are enumerated resulting in $N_{2}=11$ different partitioning solutions and the optimal observer gains are selected for the considered optimization problem OP1 or OP2. Term $P_{n_{K}}, n_{K} \in \mathscr{N}_{K}=\left[1, \ldots, N_{K}\right]$, indicates the $n_{K}$-th partitioning configurations for $K$ resulting subsystems. The sets $\mathscr{V}_{h}$ that hold the zone indices of each subsystem for all partitioning solutions for $K=2$ appear in Table I. All optimization problems are modelled using YALMIP [14] and solved using MOSEK SDP solver [15].

The detection performance for OP1 and $K=2$ is shown in Fig. 2 for all zones. The results clearly show that the partitioning choice affects the contaminant detection performance. Specifically, the resulting contaminant release rate bound $g_{i}^{z *}$ for each zone, changes depending on the partitioning solution. Partitioning $P_{9}$ can be considered as the best, since it minimizes the worst case scenario in the whole system. Similarly, $P_{1}$ can be considered as the worst, since for $\Sigma_{1}$ it displays the worst performance of all other subsystems of all possible partitioning. In particular, $P_{1}$ shows approximately $10 \%$ larger bound in 12 out of 14 zones of the building.

While the partitioning solution impacts the performance of the contaminant detection, the optimization also allows full exploitation of observer gains. The importance of jointly optimizing the partitioning configuration and observer gains is illustrated in Fig. 3 considering OP2. The term $€$ indicates an arbitrary selection of $\lambda_{i}=2, i \in \mathscr{V}$ for all zones that ensures observer stability while $L^{*}$ indicates the best observer gains as resulted from the optimization. It becomes evident that there is a major performance increase in optimizing observer gains. In particular, Fig. 3 shows that the detection performance is about 5 times worst for the arbitrary gain selection than for the optimized observer gains. Another important aspect is that the best partitioning solutions for 


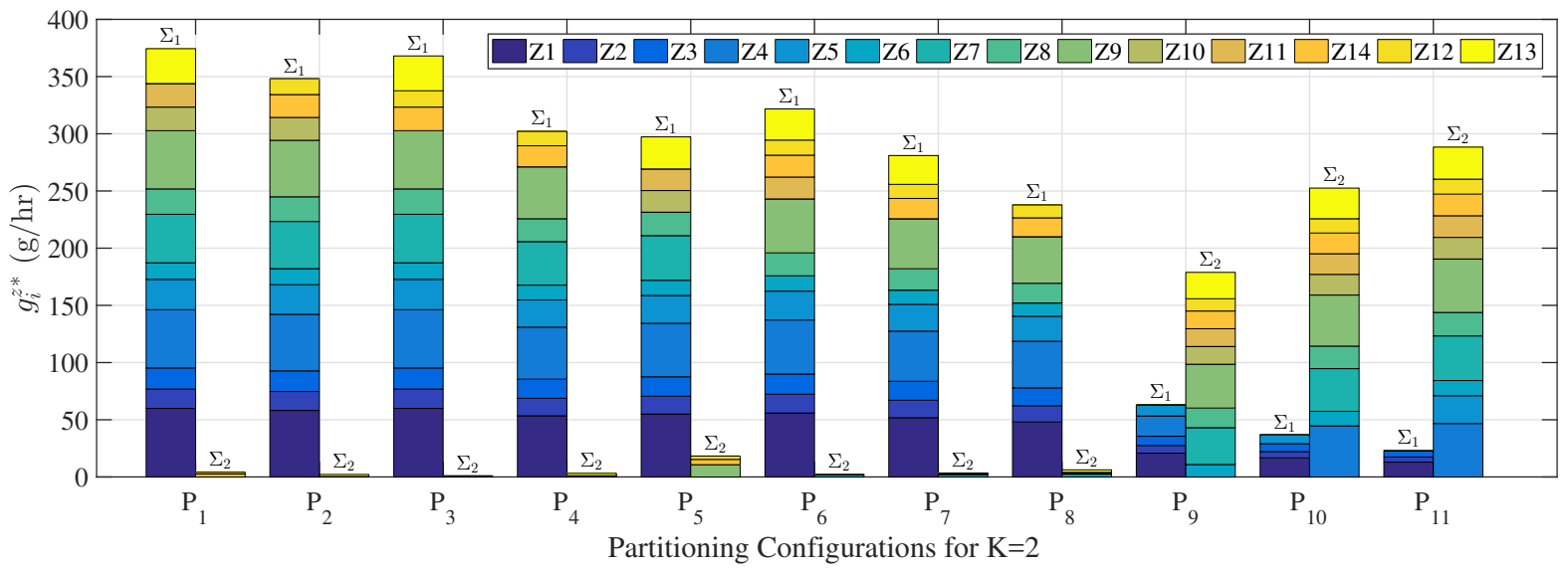

Fig. 2. Contaminant detection performance using OP1 for all possible partitioning configurations for $K=2$.

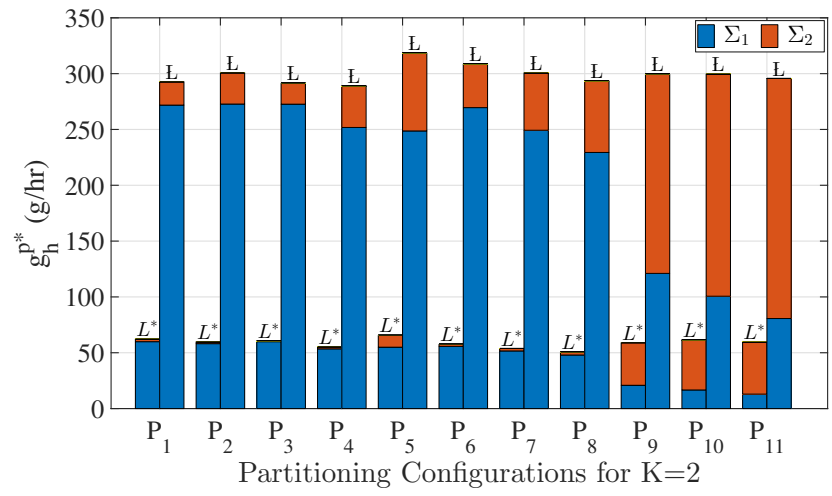

Fig. 3. Comparison of detection performance between optimal selection of observer gains $L^{*}$ and an arbitrary selection $€$ using OP2 for all possible partitioning configurations for $K=2$.

equalizing the detection performance for the subsystems are given by $P_{9}$ for both optimization objectives as shown in Fig. 2 and Fig. 3.

\section{CONCLUSIONS}

Indoor Air Quality monitoring is one of the most important properties of Intelligent Buildings that ensures the well-being of the occupants. In this work, a distributed contaminant detection approach based on a multi-zone system model is presented. The work focuses in finding the best system partitioning for the distributed approach in order to improve the contaminant detection performance. Through a detectability analysis of the approach, bounds for the lowest detectable contaminant release rate have been found, which are functions of observer gains and the system partitioning. To optimize performance, a novel approach has been developed for finding the optimal observer gains for each partitioning configuration. The results show that optimizing the observer gains leads to more than five-fold improvement compared to arbitrary gain selection and two-fold improvement for selecting the best possible partitioning solution.

Future work includes, the investigation of the partitioning selection problem for scenarios with limited number of available sensors and for the problem of contaminant isolation.

\section{REFERENCES}

[1] J. E. Braun, "Intelligent Building Systems - Past, Present, and Future," in Proceedings of American Control Conference (ACC), 2007, pp. 4374-4381.

[2] A. I. Dounis and C. Caraiscos, "Advanced control systems engineering for energy and comfort management in a building environment - A review," Renewable and Sustainable Energy Reviews, vol. 13, no. 6, pp. 1246-1261, 2009.

[3] X. Liu and Z. Zhai, "Inverse modeling methods for indoor airborne pollutant tracking: Literature review and fundamentals," Indoor Air, vol. 17, no. 6, pp. 419-438, 2007.

[4] M. P. Michaelides, V. Reppa, M. Christodoulou, C. G. Panayiotou, and M. M. Polycarpou, "Contaminant event monitoring in multi-zone buildings using the state-space method," Building and Environment, vol. 71, pp. 140-152, 2014.

[5] A. Kyriacou, M. P. Michaelides, V. Reppa, S. Timotheou, C. G. Panayiotou, and M. M. Polycarpou, "Distributed contaminant detection and isolation for intelligent buildings," IEEE Transactions on Control Systems Technology, 2017, doi: 10.1109/TCST.2017.2754986.

[6] P. Daoutidis, W. Tang, and S. S. Jogwar, "Decomposing complex plants for distributed control: Perspectives from network theory," Computers \& Chemical Engineering, vol. 114, pp. 43-51, 2018.

[7] F. Boem, R. M. Ferrari, T. Parisini, and M. M. Polycarpou, "Optimal topology for distributed fault detection of large-scale systems," in Proceedings of International Federation of Automatic Control (IFAC), vol. 48 , no. 21,2015 , pp. $60-65$.

[8] C. Ocampo-Martínez, S. Bovo, and V. Puig, "Partitioning approach oriented to the decentralised predictive control of large-scale systems," Journal of Process Control, vol. 21, no. 5, pp. 775-786, 2011.

[9] V. Reppa, M. M. Polycarpou, and C. G. Panayiotou, "Sensor fault diagnosis," Foundations and Trends in Systems and Control, vol. 3, no. 1-2, pp. 1-248, 2016. [Online]. Available: http://dx.doi.org/10.1561/2600000007

[10] V. Reppa, S. Timotheou, M. M. Polycarpou, and C. G. Panayiotou, "Optimization of observer design for sensor fault detection of nonlinear systems," in Proceedings of IEEE Conference on Decision and Control (CDC), 2017, pp. 5155-5160.

[11] L. Vandenberghe and S. Boyd, "Semidefinite programming," SIAM Review, vol. 38, no. 1, pp. 49-95, 1996.

[12] L. L. Wang, W. S. Dols, and Q. Chen, "Using CFD capabilities of CONTAM 3.0 for simulating airflow and contaminant transport in and around buildings," HVAC\&R Research, vol. 16, no. 6, pp. 749-763, 2010.

[13] G. N. Walton and W. S. Dols, "CONTAM 3.0 user guide and program documentation," National Institute of Standards and Technology Technical Report NISTIR, vol. 7251, 2010.

[14] J. Löfberg, "YALMIP : A Toolbox for Modeling and Optimization in MATLAB," in Proceedings of the CACSD Conference, Taipei, Taiwan, 2004.

[15] MOSEK ApS, The MOSEK optimization toolbox for MATLAB manual. Version 8.1., 2017. [Online]. Available: http://docs.mosek.com/8.1/toolbox/index.html 